\title{
Auditory perceptual learning for speech perception can be enhanced by audiovisual training
}

\section{Lynne E. Bernstein*, Edward T. Auer Jr., Silvio P. Eberhardt and Jintao Jiang}

Communication Neuroscience Laboratory, Department of Speech and Hearing Science, George Washington University, Washington, DC, USA

Edited by:

Claude Alain, Rotman Research

Institute, Canada

Reviewed by:

Mark T. Wallace, Vanderbilt University, USA

liro P. Jääskeläinen, Aalto University, Finland

\section{${ }^{*}$ Correspondence:}

Lynne E. Bernstein, Communication

Neuroscience Laboratory,

Department of Speech and Hearing

Science, George Washington

University, 550 Rome Hall, 810 22nd

Street, NWWashington, DC 20052,

USA.

e-mail: Ibernste@gwu.edu
Speech perception under audiovisual (AV) conditions is well known to confer benefits to perception such as increased speed and accuracy. Here, we investigated how AV training might benefit or impede auditory perceptual learning of speech degraded by vocoding. In Experiments 1 and 3, participants learned paired associations between vocoded spoken nonsense words and nonsense pictures. In Experiment 1, paired-associates (PA) AV training of one group of participants was compared with audio-only $(\mathrm{AO})$ training of another group. When tested under $\mathrm{AO}$ conditions, the AV-trained group was significantly more accurate than the AO-trained group. In addition, pre- and post-training $A O$ forced-choice consonant identification with untrained nonsense words showed that AV-trained participants had learned significantly more than $\mathrm{AO}$ participants. The pattern of results pointed to their having learned at the level of the auditory phonetic features of the vocoded stimuli. Experiment 2, a no-training control with testing and re-testing on the $\mathrm{AO}$ consonant identification, showed that the controls were as accurate as the AO-trained participants in Experiment 1 but less accurate than the AV-trained participants. In Experiment 3, PA training alternated $\mathrm{AV}$ and $\mathrm{AO}$ conditions on a list-by-list basis within participants, and training was to criterion ( $92 \%$ correct). PA training with $\mathrm{AO}$ stimuli was reliably more effective than training with AV stimuli. We explain these discrepant results in terms of the so-called "reverse hierarchy theory" of perceptual learning and in terms of the diverse multisensory and unisensory processing resources available to speech perception. We propose that early $\mathrm{AV}$ speech integration can potentially impede auditory perceptual learning; but visual top-down access to relevant auditory features can promote auditory perceptual learning.

Keywords: audiovisual speech processing, audiovisual speech perception, perceptual learning, reverse hierarchy theory, auditory perception, visual speech perception, multisensory processing, plasticity and learning

\section{INTRODUCTION}

In addition to the classically defined, high-level multisensory cortical association areas such as the superior temporal sulcus (Calvert et al., 2000; Beauchamp et al., 2004; Miller and D'Esposito, 2005; Nath and Beauchamp, 2012), multisensory processing sites have been identified at lower levels, such as primary or secondary cortical areas and the major thalamic relay nuclei (for reviews, see Foxe and Schroeder, 2005; Driver and Noesselt, 2008; Falchier et al., 2012; Kayser et al., 2012). For example, monkey studies have found visual neuronal inputs to primary auditory cortex and to the caudal auditory belt cortex (Schroeder and Foxe, 2002; Ghazanfar et al., 2005; Kayser et al., 2009). Evidence is also available for auditory neuronal inputs to primary visual cortex (Falchier et al., 2001, 2012). Extensive multisensory connectivity has led to the suggestion that all cortical operations are potentially multisensory (Ghazanfar and Schroeder, 2006).

There is no doubt that speech perception makes use of diverse multisensory cortical processing resources (Sams et al., 1991; Calvert et al., 2000; Möttönen et al., 2002; Miller and D’Esposito, 2005; Saint-Amour et al., 2007; Skipper et al., 2007; Bernstein et al., 2008a,b; Nath and Beauchamp, 2011, 2012), and that visual speech stimuli integrate with auditory stimuli under a wide range of listening conditions and for a wide range of functions. For example, when auditory speech stimuli are degraded, being able to see the talker typically leads to improved perceptual accuracy (e.g., Sumby and Pollack, 1954; MacLeod and Summerfield, 1987; Iverson et al., 1998; Ross et al., 2007; Ma et al., 2009). But even when the auditory stimuli are not degraded, visual speech stimuli can affect speech perception and comprehension. Comprehension of difficult verbal materials can be easier under audiovisual (AV) conditions (Reisberg et al., 1987); Perception in a second language can be more accurate with AV stimuli than with auditory-only stimuli (Hazan et al., 2006); and Numerous demonstrations of the McGurk effect (McGurk and MacDonald, 1976) have shown that when auditory and visual speech consonants are mismatched, perceivers often hear a consonant that is different from either the auditory or visual stimulus per se (e.g., Green and Kuhl, 1989; Sekiyama and Tohkura, 1991; Jiang and Bernstein, 2011). The study reported here addressed how training with AV speech stimuli might affect auditory perceptual learning of a type of novel degraded acoustic speech stimulus. At issue was how multisensory resources are deployed in the context of unisensory perceptual learning. 
This study focused on learning to perceive degraded acoustic speech. The spoken nonsense words that were used as stimuli were transformed by passing them through a vocoder, a signalprocessor that systematically degrades the speech (Iverson et al., 1998; Scott et al., 2000) and typically requires experience or training to achieve improved levels of perceptual accuracy (e.g., Davis et al., 2005; Scott et al., 2006; Hervais-Adelman et al., 2011). The vocoder here transformed fine-grained acoustic spectral cues, including vocal tract resonance changes that are cues to phoneme (consonants and vowels) distinctions, into coarse spectral cues by coding energy in 15 frequency bands as amplitudes of fixed-frequency sinusoids at the center frequency of each band (Figure 1). In addition, the normal speech spectrum, which falls off at approximately $6 \mathrm{~dB}$ per octave, was tilted so that amplitudes in vocoder bands were approximately equalized. Figure 1 shows spectrograms of the syllables /bE/ and /fE/ (i.e., the vowel in "bet") for the natural recorded speech (Figures 1A,C) and the vocoded speech (Figures 1B,D). The vocoding highly reduces the available acoustic information, emphasizes the second speech formant (vocal tract resonance), known to be highly informative for speech perception (Liberman et al., 1967), and reduces or omits the first and third formants, which are also important.

We hypothesized that information in visual speech stimuli can provide top-down guidance for auditory perceptual learning (Ahissar and Hochstein, 1997; Kral and Eggermont, 2007; Ahissar et al., 2008) of the cues to phoneme perception in the vocoded acoustic signals. That is, in addition to integrating with auditory speech cues during perception, visual speech stimuli were hypothesized to be able to guide auditory perceptual learning, with the result that auditory-only perception is improved more following AV than following auditory-only training. Our rationale for this hypothesis about the benefits of visual speech is that certain visual speech features can be reliably available (Bernstein et al., 2000; Bernstein, 2012), and they are correlated in real time with auditory features (Yehia et al., 1998; Jiang et al., 2002; Jiang and Bernstein, 2011). Therefore, they could help to train novel or unfamiliar vocoded auditory speech features when they are available during training. For example, /f/ and /b/ are visually distinctive (Auer and Bernstein, 1997), but the distinction between vocoded /f/ and /b/, which is available in the novel acoustic signals (see Figures 1B,D), might not be discerned without training. Training with the AV stimuli could enhance auditory perceptual learning, because the visual features that are integrated during visual perceptual processing (Bernstein et al., 2011; Bernstein, 2012) could be used to guide top-down attention to the correlated auditory cues that discriminate /f/ from /b/. In contrast, training with auditory-only stimuli contributes no additional information for learning novel cues or features, beyond what can be gleaned from merely repeating the stimulus, and the perceiver might not learn to distinguish the critical novel cues. Alternatively, early integration of auditory and visual speech features could impede auditory perceptual learning, because perception would be successful without accessing the available auditory distinctions in the vocoded stimuli.

In the study reported here, we compared auditory perceptual learning based on training with $\mathrm{AV}$ versus audio-only $(\mathrm{AO})$ speech stimuli. Because our hypothesis concerned perceptual learning of acoustic speech features, the experimental task had to preclude access to pre-existing lexical knowledge, a type of high-level representation, that could function like visual speech stimuli. Lexical knowledge itself can be a top-down source for auditory perceptual learning (Davis et al., 2005). Therefore, all of the stimuli in the study were spoken nonsense words. Auditory training was given in a paired-associates (PA) task. Participants learned paired associations between disyllabic spoken nonsense words and nonsense pictures. Training was under AV and/or AO conditions, and testing was exclusively under $\mathrm{AO}$ conditions. In addition to PA training and testing, a forced-choice identification paradigm was used to test auditory consonant identification before and after training, using stimuli that were not used in training. The consonant identification also served to test for generalization to new stimuli in a different perceptual task and to infer the level of auditory perceptual learning that was achieved. Our results show that AV training can significantly benefit auditory perceptual learning beyond $\mathrm{AO}$ training. But the details of the training protocol appear to be critically important to achieving benefit from visual stimuli, because $\mathrm{AV}$ training can also lead to poorer $\mathrm{AO}$ performance. In our General Discussion, we propose a model of how AV stimuli can guide auditory perceptual learning through top-down visual access to useful auditory distinctions; or how AV stimuli can impede auditory perceptual learning through early immediate integration of auditory and visual speech cues.

\section{MATERIALS AND METHODS EXPERIMENT 1 BETWEEN-PARTICIPANT TRAINING WITH FIXED NUMBERS OF TRAINING TRIALS}

In Experiment 1, participants were assigned to either $\mathrm{AV}$ or $\mathrm{AO}$ PA training followed by AO testing. Training in the PA task used nonsense pictures and nonsense words of the form consonantvowel-consonant-vowel-consonant (CVCVC), modeled on the phonotactics of disyllabic English words. The PA task emulated the learning of new vocabulary items. Thus, participants were required to learn at multiple levels, including the perceptual (novel acoustic transform and novel lexical word form) and the high-level associative (semantic association between word form and picture). Here, participants were tested on the number of paired associations they could demonstrate following training. If $\mathrm{AV}$-trained participants were more successful during AO testing than AOtrained participants, who had achieved equivalent performance during training, then the implication would be that the AV-trained participants learned more about the auditory stimuli. Pre- and post-training forced-choice consonant identification was tested, using an untrained set of CVCVC nonsense words. The identification measures were the number of correctly identified consonants in the three positions of the nonsense words. If differential learning occurred across the position of the consonant in the word, then the implication would be that participants learned sub-phonemic auditory features, because acoustic phonetic signals differ across segment position in a word (Stevens, 1998).

\section{Subjects}

Individuals were screened for American English as a first language, normal or corrected-to-normal vision in each eye of 20/30 or better (using a Snellen chart), and hearing ( $25 \mathrm{~dB}$ HL or better in each ear for frequencies between 125 and $8 \mathrm{KHz}$, using an Audiometrics 


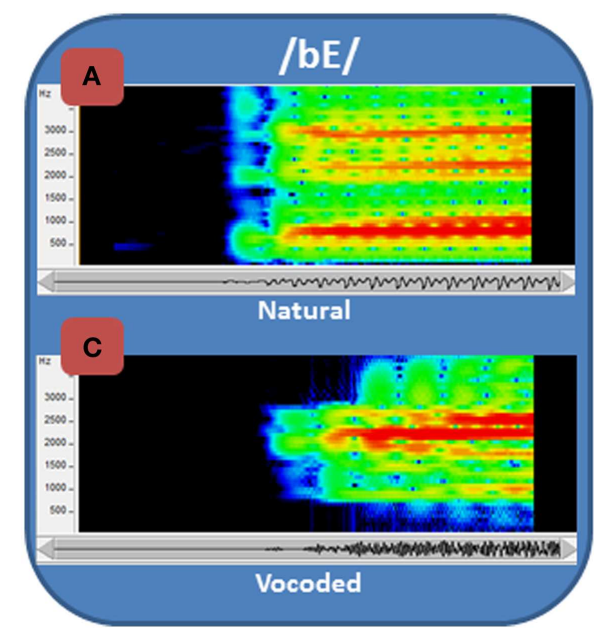

FIGURE 1 | Spectrograms of normal and vocoded speech.

Spectrograms of speech show the concentrations of energy in the spectra over time. Two speech tokens, /bE/ and /fE/ (i.e., the vowel in "bet"), are shown in spectrograms of the natural (A) and (B) recorded speech and the vocoded (C) and (D) speech. The frequency range of the spectrograms is restricted to $4 \mathrm{kHz}$, because all of the energy from the vocoder is similarly limited. The amplitudes are represented as a heat

GSI 16 audiometer with insert earphones). The experiment was carried out at two different locations, using the same equipment and procedures. At the House Research Institute (Los Angeles, CA, USA), 12 volunteers, ages $18-48$ years (mean $=30$ years), including six males, completed the experiment, and an additional five volunteers were asked to discontinue the experiment after they were mistakenly presented with non-distorted speech. At the George Washington University, 25 volunteers, ages 1930 (mean =22), including five males, completed the experiment, and an additional four dropped out due to lack of availability. In all, 18 participants completed AV training, and 19 completed AO training. They were paid $\$ 12$ per hour of testing, plus any travel expenses incurred. Subjects gave written consent. Human subject participation was approved by either the St. Vincent's Hospital Institutional Review Board (Los Angeles, CA, USA) or by the George Washington University Institutional Review Board (Washington, DC, USA).

\section{Stimuli}

Speech. The spoken CVCVC nonsense words were modeled on English phonotactics (i.e., the sequential speech patterns in English). They were visually distinct for lipreading and visually unique from real English words (i.e., the words were designed to not be mistaken as real words, if they were lipread without accompanying audio). Thus, for example, the nonsense word mucker was not included in the set, because the visual stimulus could be mistaken for the real word pucker, inasmuch as the phonemes /p, m/ are visually highly similar (Auer and Bernstein, 1997).

The process of stimulus generation was as follows. Syllables with the structure CV-, -VCV-, and -VC were extracted from the 35,000-word phonemically transcribed PhLex database (Seitz et al., 1998). Based on empirically derived phonotactic

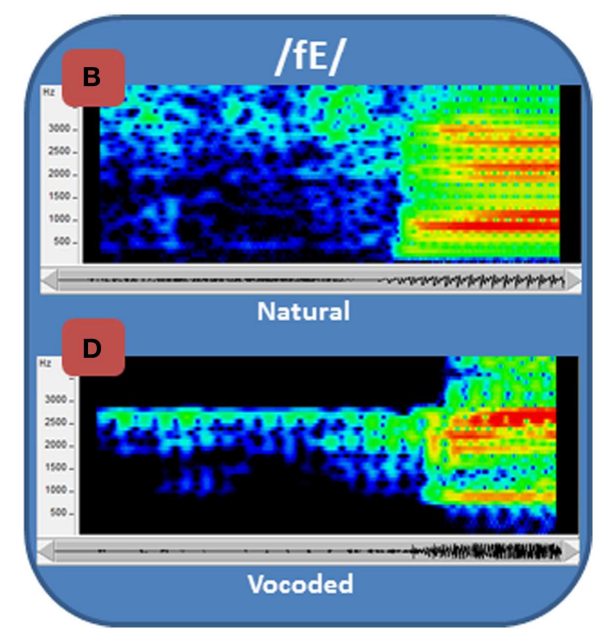

map, with red the highest amplitude and dark blue the lowest. In addition to representing the speech as the sum of sinewaves at the center of each vocoder filter (see text), the vocoder also tilted the spectrum so that it did not roll off at approximately $6 \mathrm{~dB} /$ octave, which is natural to speech. Thus, the amplitudes of the frequencies vary across the natural and the vocoded speech, in addition to the frequency ranges and spectral detail.

probabilities, a Monte Carlo simulation was used to generate 30,000 CVCVC candidate nonsense words, which were then further processed. First, existing visual phoneme confusion data were used to model the confusability of the phonemes (Auer and Bernstein, 1997; Iverson et al., 1998). Then the candidate nonsense words were computationally processed, taking into account their visual confusability with real words and other nonsense words (Auer and Bernstein, 1997). Stimuli that would have been easily confused by vision were grouped into sets, and only one CVCVC word was chosen from each set, with the requirements that (1) the final set of nonsense words would include all the English phonemes, and (2) within each CVCVC, the five phonemes would be visually distinct to a lipreader (Auer and Bernstein, 1997). These constraints implied that within a list of nonsense words, visual information should be sufficient to differentiate among items.

The female talker whose data were used to model consonant and vowel confusability was the same talker used to produce the nonsense words. She was professionally videotaped uttering the final set of 260 CVCVC words.

Stimulus lists were constructed by first ordering stimuli by initial consonant and vowel, and then dividing the list on even- versus odd-numbered items to form two lists from which items were randomly selected. Two 49-item lists were selected for the pre- and post-training consonant identification task (Table 1; see Table 2 for transcription key). Two six-item lists were selected from 12item lists for pre- and post-training practice. Six lists of 12 items for PA training and six lists of six items as new items during PA testing were selected from the remaining available words (Table 3 ).

The acoustic speech stimuli were processed through a custom realtime hardware/software vocoder (Iverson et al., 1998). The vocoder detected speech energy in thirteen 120-Hz-bandwidth bandpass filters with center frequencies every $150 \mathrm{~Hz}$ from $825 \mathrm{~Hz}$ 
Table 1 | Pre-test and post-test consonant identification lists in single-phoneme transcription format.

\begin{tabular}{|c|c|c|c|}
\hline \multicolumn{2}{|c|}{ List 1} & \multicolumn{2}{|c|}{ List 2} \\
\hline banoz & pETat & batok & $\operatorname{pod} \wedge \mathrm{n}$ \\
\hline biscg & ponRs & Bizxd & pUrIn \\
\hline brcit & pUtIl & $\mathrm{bRs} \times \mathrm{V}$ & Ribcg \\
\hline bulad & rid^t & bUnxl & rob^l \\
\hline C@GRz & $\operatorname{rot} \wedge \mathrm{k}$ & C@pRk & s@naJ \\
\hline ccrik & s@vxk & Cct IG & SIGRt \\
\hline cEmxl & sik $\wedge S$ & CEvxs & SInal \\
\hline deman & Sivab & Dumxs & sRbik \\
\hline duzxn & sRmaS & $\mathrm{fRCxl}$ & Sulak \\
\hline fRsal & suzxm & gInxz & teCig \\
\hline gIZxn & $t @ n \wedge m$ & $\mathrm{~h} @ \mathrm{n} \wedge \mathrm{p}$ & tEmaS \\
\hline h@nus & tErin & Jcrat & $\mathrm{Tib} \wedge \mathrm{n}$ \\
\hline jcrib & $\mathrm{Tis} \wedge \mathrm{p}$ & JEnap & Tufxl \\
\hline jEris & Tukad & JozIG & vesap \\
\hline junxs & VEJUd & $\mathrm{k} @ \mathrm{Cud}$ & $\mathrm{vEJxn}$ \\
\hline keTaz & $\mathrm{vob} \wedge \mathrm{n}$ & Kcrit & vomit \\
\hline kctas & $\mathrm{vRbIG}$ & $\mathrm{m} @ \mathrm{DRz}$ & vRlIs \\
\hline me JUd & Wefxn & madRz & wct $\wedge \mathrm{m}$ \\
\hline makiz & wEJxk & Mckit & wEkab \\
\hline mczin & $\mathrm{wRk} \wedge 1$ & mEros & wRlas \\
\hline mezxl & Yizxk & nECUt & yizxs \\
\hline Net $\wedge \mathrm{m}$ & yUbIg & Nobad & yUmEs \\
\hline noluz & Yusap & $\mathrm{p} @ \mathrm{Cik}$ & yut Ib \\
\hline peTan & zobIG & paJut & zoSxn \\
\hline palit & & pEluz & \\
\hline
\end{tabular}

Words are transcribed, because English orthography does not map uniquely to English phonemes. Table 2 gives the phoneme transcription key. Lists 1 and 2 were randomly selected on a per-subject basis for use in pre-test and posttest (or test, re-test) consonant identification tasks. The practice list (JUkiz, zIJXI, dISUs, JEroz, mivRd, DEkXs) was used before each test to ensure that participants understood the task.

through $2625 \mathrm{~Hz}$. Two additional filters were used to convey high frequencies. One was a bandpass filter centered at $3115 \mathrm{~Hz}$ with $350 \mathrm{~Hz}$ bandwidth and the other a highpass filter with $3565 \mathrm{~Hz}$ cutoff. The energy detected in each band was used to amplitudemodulate a fixed-frequency sinewave at the center frequency of that band (and at $3565 \mathrm{~Hz}$ in the case of the highpass filter). The sum of the 15 sinewaves comprised the vocoded acoustic signal. This acoustic transformation retained the gross spectral-temporal amplitude information in the waveform while eliminating finer distinctions such as fundamental frequency variations and eliminating the natural spectral tilt of the vocal tract resonances. Figure 1 compares /ba/ and / fa/ between the original recordings and the vocoded versions.

Nonsense pictures. Nonsense pictures in the PA task were from the "fribble" image set (Databases/TarrLab/(http://wiki.cnbc.cmu. edu/Novel_Objects)). Fribbles comprise 12 species with distinct body "core" shape and color, with 81 exemplars per specie obtained by varying the forms of each of four appendage parts. From the available images, 13 lists of 12 images each were created such that each list used three different body forms and no duplicated
Table 2 | Transcription keys for nonsense word consonants and vowels.

\begin{tabular}{|c|c|c|c|}
\hline \multicolumn{2}{|c|}{$\begin{array}{l}\text { Consonant sounds represented } \\
\text { by lower case on keyboard }\end{array}$} & \multicolumn{2}{|c|}{$\begin{array}{l}\text { Consonant sounds represented } \\
\text { by UPPER case on keyboard }\end{array}$} \\
\hline \multicolumn{4}{|l|}{ A } \\
\hline Consonant & Example & Consonant & Example \\
\hline$b$ & (b)ut & $\mathrm{C}$ & su(ch) \\
\hline$d$ & goo(d) & $\mathrm{D}$ & (th)at \\
\hline$f$ & (f)ew & G & lo(ng) \\
\hline$g$ & (g)ood & $J$ & $\operatorname{lar}(g) e$ \\
\hline h & (h)is & $S$ & (sh)e \\
\hline k & (c)an & $\mathrm{T}$ & bo(th) \\
\hline । & (l)ike & Z & u(s)ual \\
\hline $\mathrm{m}$ & (m)ore & & \\
\hline$n$ & (n)ew & \multicolumn{2}{|c|}{ consonants easily confused } \\
\hline$p$ & (p)ut & $\mathrm{D}$ & $\mathrm{T}$ \\
\hline r & (r)oom & s & S \\
\hline s & (s)ome & $g$ & G \\
\hline $\mathrm{t}$ & $\mathrm{bu}(\mathrm{t})$ & z & Z \\
\hline$v$ & gi(v)e & c & $\mathrm{J}$ \\
\hline w & (w)ill & k & \\
\hline y & (y)ou & & \\
\hline$z$ & wa(s) & & \\
\hline \multicolumn{4}{|l|}{ B } \\
\hline Vowel & Example & Vowel & Example \\
\hline a & $b(o) b$ & $@$ & $b(a) t$ \\
\hline o & $\mathrm{b}(\mathrm{oa}) \mathrm{t}$ & $E$ & $\mathrm{~b}(e) \mathrm{t}$ \\
\hline i & $\mathrm{b}(\mathrm{ea}) \mathrm{t}$ & $x$ & (a)bout \\
\hline c & b(ou)ght & u & l(u)te \\
\hline r & $b(i r) d$ & I & $b(i) t$ \\
\hline u & $\mathrm{b}(\mathrm{oo}) \mathrm{k}$ & $\wedge$ & $b(u) t$ \\
\hline
\end{tabular}

(A) Consonant transcription key. (B) Vowel transcription key. These transcription keys were used to assign a single orthographic symbol for each English consonant and vowel phoneme in the nonsense words listed in Tables 1 and 3. The consonant transcription key was used to train and test participants to carry out forced-choice consonant identification.

appendage forms, rendering the images within each list highly distinctive (Williams and Simons, 2000). No appendage was repeated across lists.

\section{Design}

Figure 2 outlines the overall design of the experiment. Participants completed pre-training consonant identification familiarization and pre-training forced-choice consonant identification. Then, on each of four different days, they completed three blocks of PA training and AO testing associated with one word list. Participants were assigned to either AV or AO training for the duration of the experiment. Following the PA training and testing, participants were tested again on AO forced-choice consonant identification.

Consonant identification familiarization procedure. The preand post-training forced-choice consonant identification involved all the English consonants. Because English orthography is not uniquely mapped to English phonemes, participants were first familiarized with the orthographic transcription system, which 
Table 3 | Word lists for paired-associates task. Lists 1-4 were used in Experiment 1.

\begin{tabular}{|c|c|c|c|c|c|c|c|}
\hline Training list 1 & Test list 1 & Training list 2 & Test list 2 & Training list 3 & Test list 3 & Training list 4 & Test list 4 \\
\hline s ICUd & s ICUd & mITak & mITak & hIluz & hIluz & kizxl & Kizxl \\
\hline pcrid & pcrid & lRman & lRman & Cudxk & Cudxk & wEs Ik & wEsIk \\
\hline CRfIG & CRfIG & Sczxn & Sczxn & bUran & bUran & Bincl & Bincl \\
\hline wInct & wInct & Bodut & Bodut & Jobxt & Jobxt & Pcgxs & Pcgxs \\
\hline kUmxl & kUmxl & Ridap & Ridap & $m e f i s$ & mefis & TuSxz & TuSxz \\
\hline hUbIG & hUbIG & zEriC & zEriC & kcrac & kcraC & s@bad & s@bad \\
\hline digaz & SEsxl & pIDRz & $p E t \wedge f$ & tEfRk & zEnop & Yupan & $m @ d \wedge v$ \\
\hline IIZXS & bozEn & WRs IG & f@Jxs & Ncrim & $\mathrm{dik} \wedge \mathrm{p}$ & hob $\wedge k$ & SRfxn \\
\hline mcTxs & JovRs & $k @ f R t$ & viw^s & rilın & $\mathrm{yUS} \wedge \mathrm{k}$ & dISxp & l@kat \\
\hline tETan & metuT & TEmat & nIsxJ & TIfxs & rIZxI & vIpxd & zESxm \\
\hline rip^J & fctab & dib^J & JUkiz & fICUt & Lctak & $\mathrm{m} @ \mathrm{JxV}$ & CIlxz \\
\hline Yulat & D@ zxk & sEJud & wEsXJ & Sedxz & wevxt & Nupis & $\mathrm{fEkRz}$ \\
\hline Training list 5 & Test list 5 & Training list 6 & Test list 6 & & & Practice list 1 & Practice list 2 \\
\hline zudxn & Zudxn & mEzud & mEzud & & & fISxb & hRsak \\
\hline wizcg & Wizcg & bikud & bikud & & & ballot & pEJun \\
\hline menad & m@nad & SIzxV & SIzxV & & & yutin & buris \\
\hline$C \Theta z x d$ & $\mathrm{C} @ \mathrm{zxd}$ & hivan & hivan & & & mRsaC & JEroz \\
\hline pincg & Pincg & vid^n & $\operatorname{vid} \wedge \mathrm{n}$ & & & DEkxs & pEvxk \\
\hline y@pat & y@pat & JIfxl & JIfxl & & & bon^f & Mizcl \\
\hline b@GIt & ketup & nimat & pErit & & & $\mathrm{z} \operatorname{ErIp}$ & dISus \\
\hline hozIk & gIsan & pasIk & naSis & & & ripEs & dipcs \\
\hline lipRt & h@Jus & rigab & $\mathrm{kRCxm}$ & & & hISxd & vRpad \\
\hline fcris & Sigak & tcrab & gEsak & & & hon^t & mivRd \\
\hline nopiz & Fonab & $k @ p I G$ & wimun & & & hImut & $d I s \wedge f$ \\
\hline rik^f & $r E m R z$ & wilus & zIJxl & & & $p @ f \times J$ & wEvRz \\
\hline
\end{tabular}

Practice List 1 was used to familiarize participants with the task. Lists 1-3 were used for AO training and testing, and Lists 4-6 for AV training and AO testing. Practice List 2 was presented AO, and Practice List 1 was presented AV. Test lists always show that the first six words in the list were carried into testing and six new words were substituted for six trained words. (Table 2 gives the transcription key for phoneme mappings.)

was compatible with single-character keyboard entry. An answer key (the consonants listed in Table 2), also available during testing, was used to explain the orthographic system. During familiarization, participants filled out two self-scored worksheets, one with the key available and one without. The participants' task was to transcribe 48 consonants in real English words while looking at the key and then 71 consonants in real words without looking at the key. A six-item practice test was randomly selected from two practice lists. All the participants were able to use the orthographic transcription system.

Pre-and post-training test procedure. Audio-only forced-choice consonant identification was carried out with CVCVC nonsense words. On each trial, following presentation of a stimulus, a response string of the form "______ " appeared on the monitor, and the participants typed, in order, the three consonants that they had perceived in the AO spoken stimulus. They were instructed to guess when necessary. Only characters from the response set were displayed in the response string. It was possible to correct a response, and use of the enter key completed the trial. No feedback was given for the correctness of the responses. Different test lists were assigned across pre- and post-training testing, and list order was counter-balanced across participants.
Paired-associates training procedure. Figure 3 outlines the design of a PA training trial. During training, the participant's task was to learn, with feedback over repeated presentations, lists of individual associations between 12 fribble images and 12 CVCVC vocoded spoken nonsense words. In Figure 3, an AV training trial is shown in the left column and an AO training trial is shown in the right column. Each trial began with a computer-monitor display of the 12-fribble image matrix (three rows of four columns, with image position within the matrix randomly selected on a trial-by-trial basis). During AV training, a video of the talker was played in synchrony with the spoken audio, and during AO training, a single still image of the talker's face was displayed on the monitor during audio presentation. The talker was presented on a different monitor than the fribble matrix monitor, and a large arrow appeared on the bottom of the fribble monitor pointing left to remind the participant to focus attention on the talker. The participant used the computer mouse to choose a fribble image following the speech stimulus. Feedback was given by outlining the correct fribble in green and an incorrect choice in red. After a short interval, the speech stimulus was always repeated, while the fribble images and borders remained unchanged. A training block comprised two repetitions of the 12 paired associations in pseudorandom order. Prior to the first training list in each 


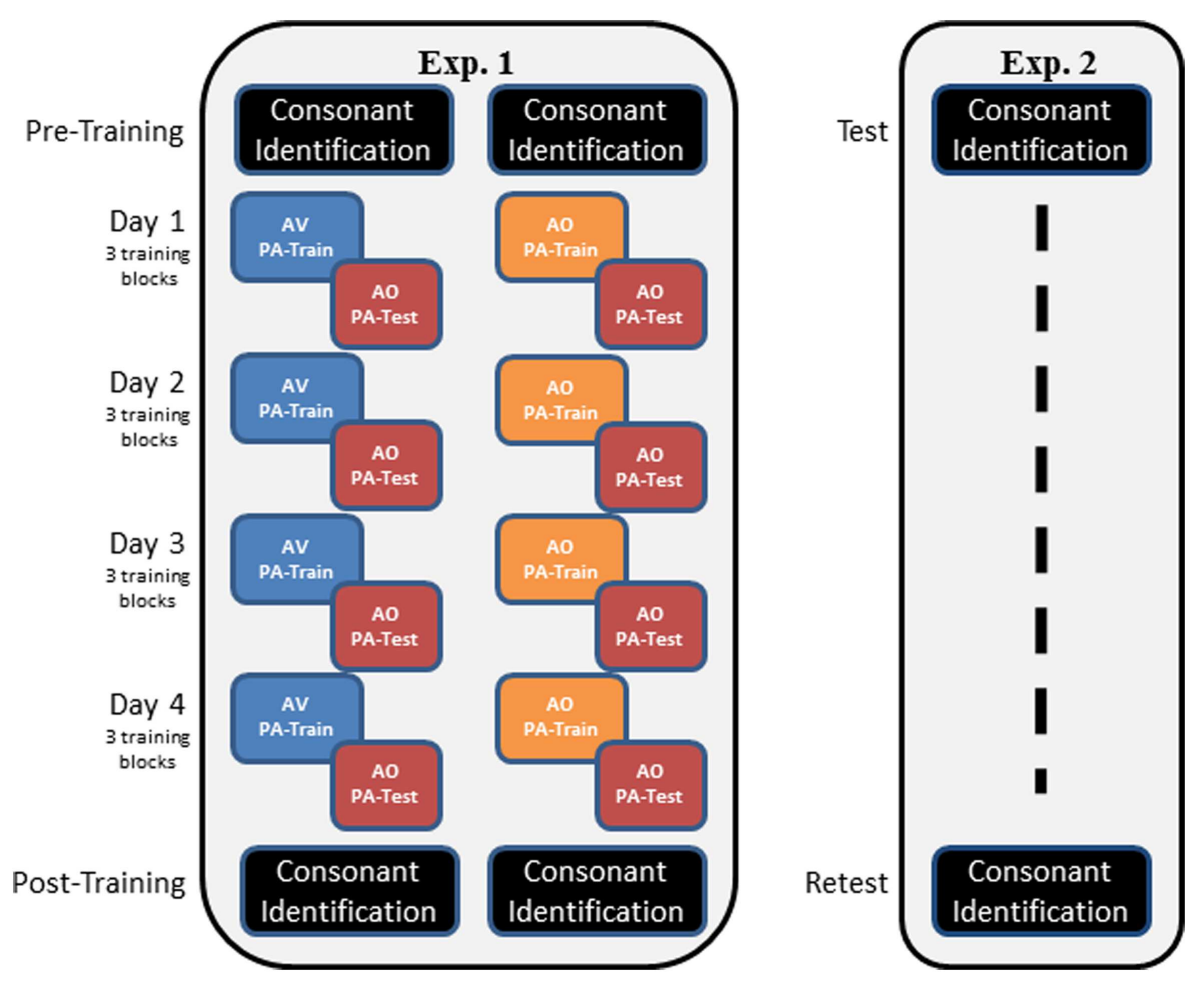

FIGURE 2 | Overall designs of Experiments 1 and 2. In Experiment 1, participants carried out pre-training consonant identification, followed by either $A V$ or $A O$ training on four stimulus lists, with $A O$ tests on each list. Training of three blocks per list was carried out on a separate day for each list. Post-training, participants were tested again on AO forced-choice consonant identification. In Experiment 2, participants were tested only on forced-choice consonant identification on two different days (test, re-test). The two administrations of the forced-choice consonant identification used different stimulus lists. condition (AV or $\mathrm{AO})$, participants were given practice with one block of six trials.

Paired-associate testing procedure. paired-associates testing immediately followed training. The testing procedure was the same as that of PA training, except the stimuli were always AO, no feedback was given, the stimulus was not repeated during the trial, and each response triggered the next trial. Six of the trained spoken words and all 12 of the fribble images were used for testing. The associations for the six retained words were unchanged. Six new nonsense words were paired with the fribble images of the discarded words. A testing block comprised, in pseudorandom order, one presentation of the 12 stimuli, and three blocks were presented. The test score was the proportion of correct paired associations of trained words.

Apparatus. Audiovisual CVCVC tokens were digitized, edited, and conveyed to digital video disk (DVD) format. The acoustic waveforms were vocoded in real time, and the audio stimuli were output at a calibrated $65 \mathrm{~dB}$ A-weighted sound pressure level (SPL) using a JBL LSR6325P-1 loudspeaker. Participants were tested in an Industrial Acoustics Company (IAC) double-walled sound-attenuating booth using a standard computer interface that included a $51 \mathrm{~cm}$ LCD monitor, and a $35.6 \mathrm{~cm}$ Sony PVM-14N5U NTSC video monitor for display of speech video from the DVD.
Monitors were located about $1 \mathrm{~m}$ from the participant's eyes, so that the computer-monitor subtended a visual angle of $23.1^{\circ}$ horizontally and 17.3 vertically with the 12 fribble matrix filling the monitor. The visual speech was displayed on the NTSC monitor with the talker's head subtending visual angles of $3.9^{\circ}$ horizontally and 5.7 vertically. Custom software was used to run the experiment.

Analyses. In order to stabilize the variance of proportion correct scores, the arcsin transformation, $X^{1}=\sin ^{-1} \sqrt{X}$ was computed, where $X$ was the proportion correct score computed over the appropriate set of trials. All analyses were also conducted in parallel on untransformed scores, and all of the parallel analyses agreed. Statistics are reported on the arcsin transformed data, but tables, means, and figures are untransformed to facilitate interpretation.

\section{Results and discussion}

Paired-associates training. Initial inspection of the training and testing data showed there to be wide individual variation. There were participants who were unable to learn associations to an acceptably high-level of accuracy within the three training blocks. In order to assure that a relatively similar level of PA learning had taken place across training conditions, the criterion of at least $75 \%$ correct on the third training block was set for use of a participant's data. That is, we chose to remove the data sets obtained 


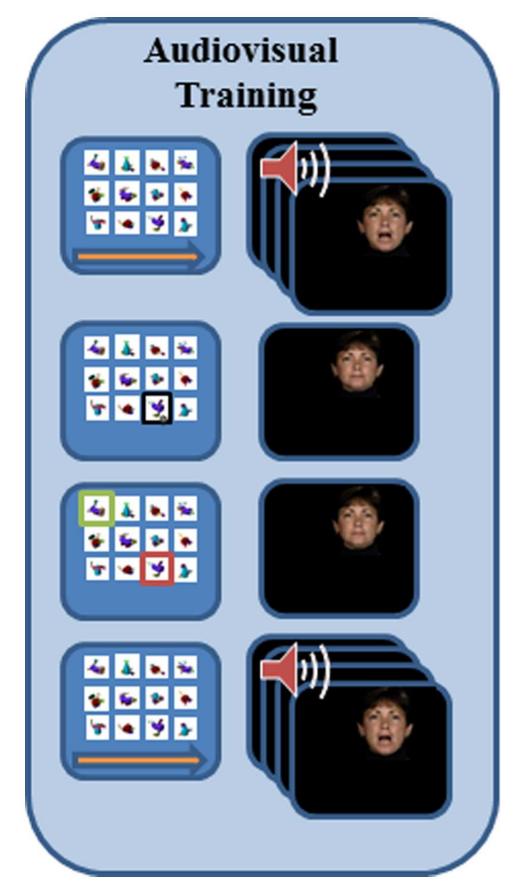

FIGURE 3 | Trial structure for paired-associates training. A speech stimulus was presented, followed by the participant's response selection, followed by feedback and a repetition of the speech stimulus. Each panel depicts the screen showing the fribble images side-by-side with the video monitor

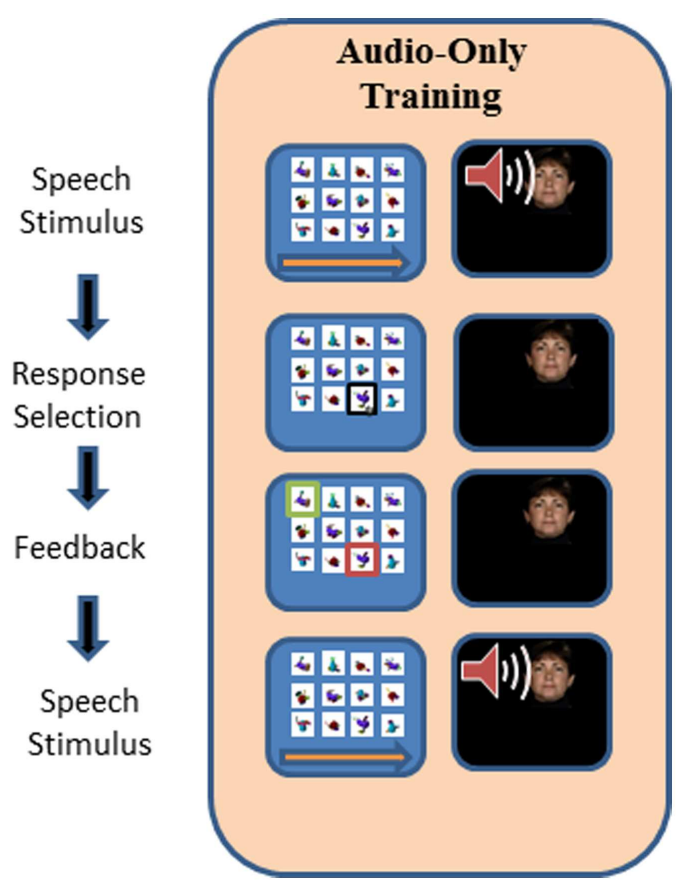

showing the talker. The trial structure for $\mathrm{AV}$ and $\mathrm{AO}$ training followed the same sequence, except that during AV training the video was played synchronously with the audio, and during $A O$ training a still neutral face was played during the audio. from participants who appeared to have difficulty learning associations per se. This criterion removed data from 10 participants from analyses. An additional participant was dropped because of scoring $6 \%$ correct on the test of one list, deviating greatly from typical test performance $($ mean $=94 \%$, minimum $=67 \%$, maximum $=100 \%)$. The analyses reported henceforth are on the data from 25 participants, 12 in the AV-trained group and 13 in the AO-trained group.

To examine performance during training, scores were submitted to RMANOVA with the within subjects factors of training list (1-4) and training block (1-3), and the between-subjects factor of training group (AO-trained, AV-trained). Importantly, no evidence was obtained for a reliable main effect or interaction with training group. Reliable main effects were obtained for training list $F(3,69)=19.26, \mathrm{MSE}=0.49, p<0.001, \eta_{p}^{2}=0.46$, and training block, $F(2,46)=651.09$, MSE $=14.41, p<0.001, \eta_{p}^{2}=0.97 . \mathrm{A}$ significant interaction between list and block (see Table 4), $F(6$, $138)=6.77, \mathrm{MSE}=0.08, p<0.001, \eta_{p}^{2}=0.23$, was also obtained. Table 4 shows that, with experience, learning was faster.

\section{Paired-associates test results}

The critical question was whether the AV-trained participants were more accurate than AO-trained participants when both were tested with AO stimuli. The proportion correct PA test scores based on three repetitions of each of the six trained items was computed. The values were submitted to RMANOVA with the within subject factor of training list (1-4) and the between subject factor training condition $(\mathrm{AO}, \mathrm{AV})$. A main effect of training condition, $F(1$,
Table 4 | Experiment 1 training scores as a function of list and block.

\begin{tabular}{llll}
\hline & Block 1 & Block 2 & Block 3 \\
\hline List 1 & $31(2.0)$ & $76(3.3)$ & $95(1.3)$ \\
List 2 & $42(2.2)$ & $90(2.0)$ & $98(0.8)$ \\
List 3 & $49(2.5)$ & $93(1.6)$ & $96(1.2)$ \\
List 4 & $51(2.1)$ & $91(1.8)$ & $97(1.0)$
\end{tabular}

The means are presented with the standard error of the mean in parenthesis.

$23)=7.619, \mathrm{MSE}=0.36, p<0.05, \eta_{p}^{2}=0.25$, was obtained. The $\mathrm{AV}$-trained participants had higher $\mathrm{AO}$ test scores $(97 \%$ correct test scores, $\mathrm{SE}=1.4)$ than did the AO-trained participants $(92 \%$ correct test scores, $\mathrm{SE}=1.4)$. No other effects were reliable. The responses to the six untrained words that were presented during testing were also checked for accuracy, and the scores were very low.

\section{Pre- and post-training results}

Forced-choice consonant identification data were collected preand post-training on independent lists of AO nonsense words. Proportion correct identification scores for consonants in initial, medial, and final position were computed separately on preand post-training data. Scores were submitted to RMANOVA with within-subject factors of time of testing (pre- versus posttraining), consonant position (initial, medial, and final), and between-subjects factor group (AV-trained, AO-trained). The main effects of time of testing, $F(1,23)=141.08, \mathrm{MSE}=0.98$, 
$p<0.001, \eta_{p}^{2}=0.86$, and of consonant position, $F(2,46)=49.22$, $\mathrm{MSE}=0.28, p<0.001, \eta_{p}^{2}=0.68$, were both reliable

The interaction between time of testing and group was reliable, $F(1,23)=8.54, \mathrm{MSE}=0.06, p<0.05, \eta_{p}^{2}=0.27$. The AV-trained participants had lower pre-training forced-choice consonant identification scores and higher post-training scores (AV-trained pre $32 \%$ correct, post 50\% correct; AO-trained pre 35\% correct, post $47 \%$ correct), improving on average by $18 \%$ points. The AO-trained participants group improved their scores on average by $12 \%$ points. Because the two groups were different at pretraining, as well as post-training, post-training - pre-training gain scores were computed and submitted to an independent samples t-test. The gains obtained by the AV-trained group were significantly larger than the gains of the AO-trained group, $t(23)=2.91$, $p<0.05$ (see Figure 4).

The interaction between time of testing and consonant position was reliable, $F(2,46)=4.49, \mathrm{MSE}=0.02, p<0.05, \eta_{p}^{2}=0.16($ see Table 5). Post hoc tests with RMANOVA using the results for the individual consonant positions (initial, medial, and final) revealed that the magnitude of the difference in accuracy between initial and medial consonants was larger post-training than pre-training, $F(1,24)=7.45, \mathrm{MSE}=0.07, p<0.05, \eta_{p}^{2}=0.24$, as was the difference between final and medial consonants, $F(1,24)=5.67$, $\mathrm{MSE}=0.07, p<0.05, \eta_{p}^{2}=0.19$. That is, the biggest perceptual learning gains were obtained for medial consonants (see Figure 4). $\mathrm{AV}$-trained participants gained $24 \%$ points accuracy for medial consonants, and $\mathrm{AO}$-trained participants gained $17 \%$ points.

\section{EXPERIMENT 2 NO-TRAINING CONTROL}

In Experiment $1, \mathrm{AV}$ training resulted in better $\mathrm{AO}$ paired association learning and more accurate forced-choice consonant identification than did AO training. However, the design could not be used to conclude that all gains on the forced-choice consonant identification task were due to training. Therefore, a control

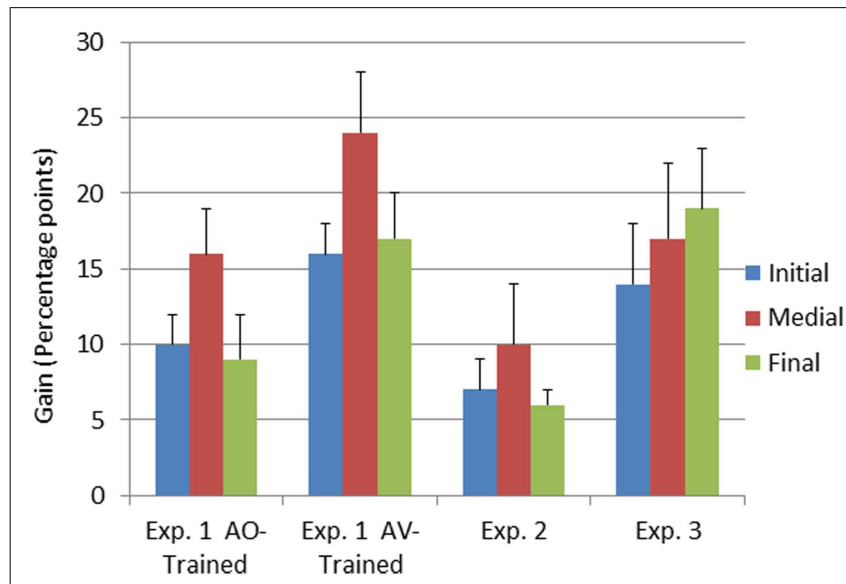

FIGURE 4 | Pre-to-post-training gain scores as a function of experiment and consonant position. Gain scores represent the means of the arithmetic difference between first and second forced-choice consonant identification test scores obtained in Experiments 1-3. The error bars represent 1 SE of the mean. Results are shown separately for the three consonant positions in the CVCVC stimuli. experiment was conducted in which the forced-choice consonant identification task was administered twice but without intervening training.

\section{Materials and methods}

Subjects. Ten volunteers, aged 22-48 years of age, two male, participated in the experiment. The criteria for inclusion were the same as in Experiment 1.

Procedure. Only the brief AO consonant familiarization procedure, practice, pre-training (test), and post-training (re-test) consonant identification tests were administered (Figure 2). The time between test and re-test ranged from 3 to 16 days (mean $=8.1$ days). The procedures for administering the forcedchoice consonant identification were the same as in Experiment 1 .

Results and discussion. The test and re-test forced-choice consonant identification data were submitted to RMANOVA with within-subject factors of time of testing (test, re-test) and consonant position (initial, medial, final). The main effects of time of testing, $F(1,9)=24.49$, MSE $=0.10, p<0.05, \eta_{p}^{2}=0.73$, and of consonant position, $F(2,18)=32.55$, $\mathrm{MSE}=0.13, p<0.001$, $\eta_{p}^{2}=0.78$, were reliable. There were no reliable interactions. Identification accuracy increased from test $(36 \%$ correct, $\mathrm{SE}=2.7)$ to re-test $(44 \%$ correct, $\mathrm{SE}=3.1)$. Linear contrasts revealed that accuracy differed among all three consonant positions (initial $=34 \%$, $\mathrm{SE}=2.7 ;$ medial $=49 \%, \mathrm{SE}=3.6$; final $=37 \%$ correct, $\mathrm{SE}=2.7$ ) (see Table 5).

Consonant identification gain scores from Experiments 1 and 2 (Figure 4) were submitted to RMANOVA with the between subject factor training group (AO-trained and AV-trained from Experiment 1 and no-training control from Experiment 2) and the within subject factor consonant position (initial, medial, final).

Table 5 | Pre-training and post-training forced-choice consonant identification scores across experiments as a function of consonant position.

\begin{tabular}{cllll}
\hline & & \multicolumn{3}{c}{ Consonant Position } \\
\cline { 3 - 5 } & & Initial & Medial & Final \\
\hline Experiment 1 & & & & \\
AO training & Pre- & $30(1.7)$ & $41(3.7)$ & $34(2.5)$ \\
& Post- & $40(2.2)$ & $58(3.2)$ & $43(3.0)$ \\
AV training & Pre- & $27(1.7)$ & $37(3.9)$ & $30(2.6)$ \\
& Post- & $43(2.3)$ & $61(3.3)$ & $47(3.1)$ \\
Experiment 2 & Test & $31(3.2)$ & $44(3.4)$ & $34(2.5)$ \\
& Re-test & $37(2.5)$ & $54(4.7)$ & $40(3.1)$ \\
Experiment 3 & Pre- & $31(2.4)$ & $47(4.4)$ & $34(2.6)$ \\
& Post- & $46(4.4)$ & $64(4.5)$ & $53(4.1)$
\end{tabular}

The tabled values are the percent correct means and standard error of the means in parentheses for each of the consonant positions in the CVCVC stimuli. In Experiments 1 and 3, the scores were obtained pre- and post-training. In Experiment 2 , the scores were obtained without intervening training (test, re-test). 
Training group was a reliable factor, $F(2,32)=10.42, \mathrm{MSE}=0.13$, $p<0.001, \eta_{p}^{2}=0.83$. Pair-wise comparisons between AO-trained (Experiment 1), AV-trained (Experiment1), and the no-training control (Experiment 2) showed that AV-trained participants had significantly higher forced-choice consonant identification gain scores than controls (see Figure 4) $(p<0.05)$. But gain scores of Experiment 1 AO-trained participants were not reliably different from those of the no-training controls. Thus, across experiments, only the AV-trained participants demonstrated auditory perceptual learning that was more successful than merely participating in a test-re-test consonant forced-choice identification task.

Consonant position was reliable in the comparison across groups, $F(2,64)=4.37$, $\mathrm{MSE}=0.04, p<0.05, \eta_{p}^{2}=0.12$. Pairwise comparisons revealed that medial pre-to-post gain scores differed from initial and final gain scores (initial $=11.6 \%, \mathrm{SE}=1.3$; medial $=17.6 \%, \mathrm{SE}=2.3$; final $=11.2 \%, \mathrm{SE}=2.7 ; p<0.05)$.

\section{EXPERIMENT 3 WITHIN-PARTICIPANT AUDIOVISUAL AND AUDITORY-ONLY TRAINING}

In Experiment 3, a modified training protocol was carried out in order to test whether the AV training advantage in Experiment 1 would be reliable under a different training protocol. Training followed that of Experiment 1, except that participants were trained until they reached the criterion of $92 \%$ correct within a training block and list. Also, AV and AO training conditions were alternated across lists, and six lists were trained (Figure 5).

\section{Materials and methods}

Subjects. Fifteen participants were recruited and started the experiment. The criteria for inclusion in the experiment were the same as in Experiment 1. Two dropped out due to difficulty learning the paired associations. The 13 who completed testing were ages $21-51$ years $($ mean $=28$ years $)$, with two males.

Procedures. Mixed $\mathrm{PA} \mathrm{AV}$ and $\mathrm{AO}$ training was given with counter-balanced initial condition and six lists total (AO, AV, AO, $\mathrm{AV}, \mathrm{AO}, \mathrm{AV}$, or $\mathrm{AV}, \mathrm{AO}, \mathrm{AV}, \mathrm{AO}, \mathrm{AV}, \mathrm{AO}$ ) (see Figure 5). Testing was always AO. Every list of paired associations was trained until the participant scored at least $92 \%$ correct. Then, in the same session, the corresponding AO test was administered. Participants were permitted to train on more than one list per session. The forced-choice consonant identification test was administered preand post-training as in Experiment 1.

\section{Results}

Paired-associates training. The number of training trials to achieve the $92 \%$ correct criterion was submitted to RMANOVA with the within subjects factors of training condition $(\mathrm{AO}, \mathrm{AV})$ and list (first, second, third). The main effect of list, $F(2,24)=4.85$, $\mathrm{MSE}=1602.46, p<0.05, \eta_{p}^{2}=0.29$, was the only factor that reached significance. Pair-wise comparisons indicated that, across training condition, more trials ( mean $=76.6$, SE 6.16) were needed to reach criterion on the first list than on the second ( mean $=64.6$, SE 5.18) and third (mean $=61.8$, SE 5.74) $(p<0.05)$, and the latter two did not differ.

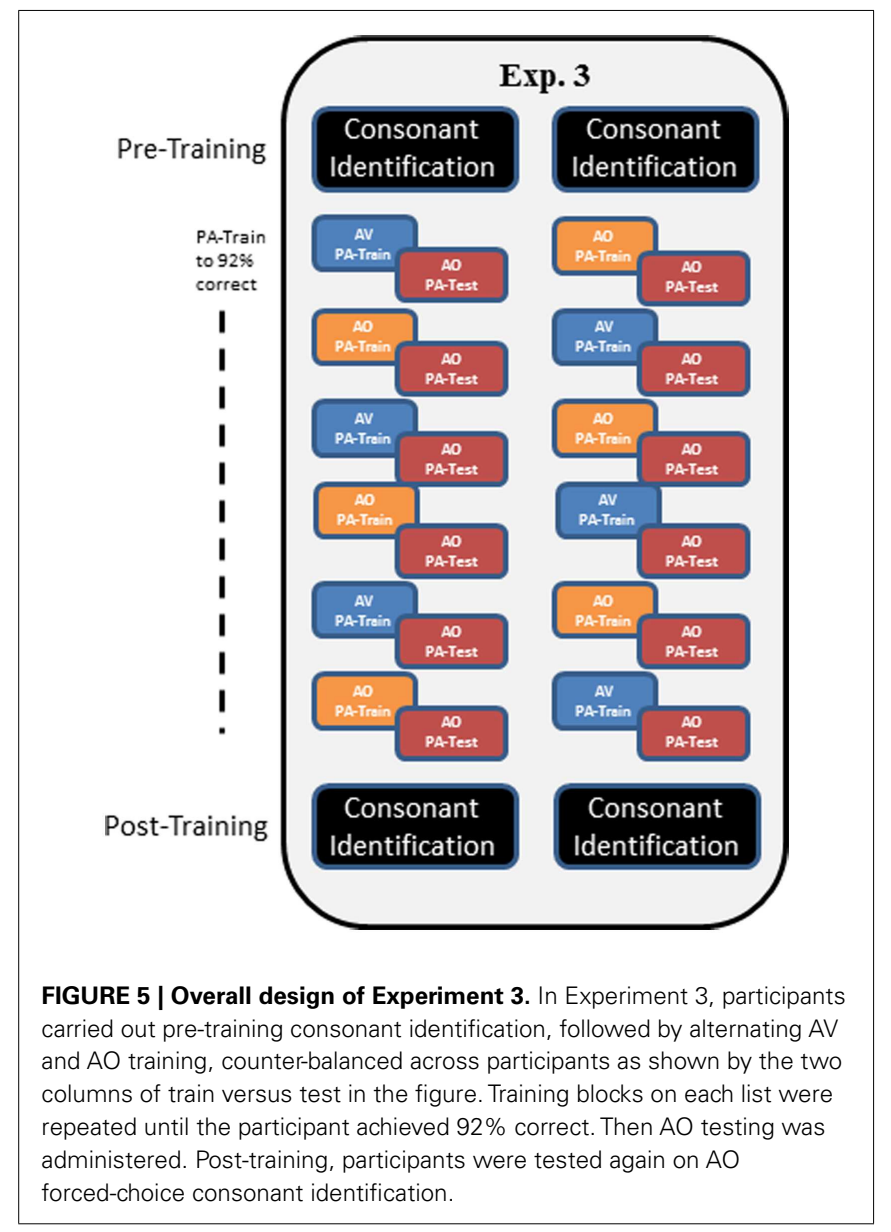

The mean accuracy scores over the blocks to criterion within a list were also submitted to RMANOVA with the within subjects factors of training condition $(\mathrm{AO}, \mathrm{AV})$ and list (first, second, third). Again, the main effect of list, $F(2,24)=14.15$, $\mathrm{MSE}=0.04, p<0.001, \eta_{p}^{2}=0.54$, was the only significant factor. Pair-wise comparisons indicated that the first list was less accurate (mean $=66.5, \mathrm{SE}=1.5)$ than the second (mean $=71.6, \mathrm{SE}=1.8$ ), which was less accurate than the third (mean $=73.9, \mathrm{SE}=1.2$; $p<0.05)$.

Paired-associates test results. The PA test results were submitted to RMANOVA with within subject factors of training condition (AO, $\mathrm{AV}$ ) and list (first, second, third). The main effect of training condition was the only significant effect, $F$ $(1,12)=8.44, \mathrm{MSE}=0.25, \mathrm{p}<0.05, \eta_{p}^{2}=0.41$. AO-trained PA test scores were higher $(94.0 \%$ correct mean test score, $\mathrm{SE}=1.8)$ than AV-trained PA test scores $(88.9 \%$ correct mean test score, $\mathrm{SE}=2.5)$.

In Experiment 1, AV PA training resulted in higher AO test scores $(97 \%$ correct test scores, $\mathrm{SE}=1.4)$ than did AO training $(92 \%$ correct AO test scores, $\mathrm{SE}=1.4)$. To compare PA test scores across Experiments 1 and 3 (which had different designs), we pooled test scores within subject separately for AV-and AO-trained lists in each experiment. The results showed that AV training in Experiment 1 was significantly more effective than in Experiment 
3, $t(23)=2.78, p<0.05$. But the AO scores were not different across experiments.

The discrepancy in PA results across Experiments 1 and 3 might have been related to the different criteria for learning that was used to accept data. In Experiment 1, a performance criterion of 75\% correct on the third training block for each list was used for inclusion of data. This resulted in dropping 10 out of 36 participants (another one was dropped for an exceptionally low AO test score on trained stimuli). In Experiment 3, two participants were unable to learn the PA stimuli to criterion of $92 \%$ correct. However, if we had imposed the $75 \%$ correct criterion on the third training block in Experiment 3, 4 out of 13 participants would have failed, which is a comparable proportion to that of Experiment 1 . Thus, the results across experiments seem unlikely to be related to group differences in ability to learn paired associations.

Pre- and post-training consonant identification. Forced-choice consonant identification scores were submitted to RMANOVA with the within subjects factors of time of testing (pre- versus post-training) and consonant position (initial, medial, final). The main effects of time of testing, $F(1,12)=15.83$, MSE $=0.68$, $p<0.05, \eta_{p}^{2}=0.57$, and of consonant position, $F(2,24)=38.99$, $\mathrm{MSE}=0.23, p<0.001, \eta_{p}^{2}=0.77$, were reliable (see Figure 4 and Table 5). The interaction between time of testing and consonant position was not reliable. Consonant identification accuracy increased from pre- $(37 \%$ correct, $\mathrm{SE}=2.7)$ to post-training $(54 \%$ correct, $\mathrm{SE}=4.1)$. Linear contrasts revealed that accuracy differed between all three positions (initial $=38 \%, \mathrm{SE}=2.7$; medial $=56 \%, \mathrm{SE}=3.8$; final $=43 \%$ correct, $\mathrm{SE}=2.7$ ).

\section{GENERAL DISCUSSION}

The results of this study suggest that AV training can promote auditory perceptual learning of novel, vocoded speech more effectively than AO training. But the training procedure affects perceptual learning outcomes. In Experiment 1, PA training was carried out with disyllabic spoken nonsense words and nonsense pictures. Participants were assigned to learn the associations with either AV or AO speech stimuli within a fixed number of trials. AV training was significantly more effective than $\mathrm{AO}$ training, as measured by testing how well the paired associations could be identified with AO stimuli. Pre- and post-training forced-choice consonant identification was also administered $\mathrm{AO}$ with untrained sets of disyllabic spoken nonsense words. On this task also, AV-trained participants were more accurate than AO-trained participants. Perception of medial consonants was significantly affected by AV training. AV-trained participants gained $24 \%$ points accuracy for medial consonants, and AO-trained participants gained 17\% points. In Experiment 2, a control experiment, participants were tested twice in the forced-choice consonant identification paradigm but without intervening training or feedback of any kind. Their re-test scores were significantly higher than their initial scores. The consonant identification scores were then compared across Experiments 1 and 2. The comparison showed that AOtrained participants in Experiment 1 were no more accurate on consonant identification than re-tested participants in Experiment 2. In contrast, AV-trained participants in Experiment 1 were more accurate than re-test participants in Experiment 2. Experiment 3 was carried out using PA training that alternated between AV and $\mathrm{AO}$ conditions on a list-by-list basis (mixed training). Training was to a $92 \%$ correct criterion, and two more lists were trained than in Experiment 1. Lists tested after AO training resulted in significantly higher AO PA scores than lists tested after AV training. Test scores on the paired associations were compared across Experiments 1 and 3. AV-trained participants in Experiment 1 were significantly more accurate $(97 \%$ correct) than participants in Experiment 3 following AV training (88.9\% correct). AO-trained participants in Experiment 1 performed similarly to participants in Experiment 3 following AO training (Experiment 1, 92\% and Experiment 3, $94.0 \%$ correct).

\section{REVERSE HIERARCHY THEORY FOR MULTISENSORY SPEECH PROCESSING}

The results of Experiment 1 suggest that multisensory stimuli can be used for improving unisensory perceptual learning. But the results of Experiment 3 suggest that multisensory stimuli can also impede unisensory perceptual learning. A theory of perceptual learning (Goldstone, 1998) is needed to explain these discrepant results. We have adopted the reverse hierarchy theory (RHT) of perceptual learning (Ahissar and Hochstein, 1997; Ahissar et al., 2008), because it attempts to explain perception and perceptual learning within the context of neural processing.

The hierarchy in RHT refers to the organization of visual and auditory sensory-perceptual pathways (Felleman and Van Essen, 1991; Kaas and Hackett, 2000). Although sensory-perceptual pathways are not strictly hierarchical, their organization is such that higher-levels show selectivity for increasingly complex stimuli combined with an increasing tolerance to stimulus transformation and increasing response to perceptual category differences (Hubel and Wiesel, 1962; Ungerleider and Haxby, 1994; Logothetis and Sheinberg, 1996; Zeki, 2005).

According to RHT, immediate perception relies on established high-level representations in the bottom-up sensory-perceptual pathway. When a new perceptual task needs to be carried out, naïve performance is initiated on the basis of immediate highlevel perception. However, if the task cannot be readily performed with the existing mapping of low-level to high-level representations, and/or if there is incentive to increase the efficiency of task performance, then perceptual learning is needed. According to RHT, perceptual learning is the access to and remapping of lower-level input representations to higher-level representations. To carry out the remapping, perceptual learning involves "perception with scrutiny." That is, a backward search must be initiated to access the representational level of the information needed to carry out the perceptual task. A new mapping can then be made. Mapping changes can occur in both convergence and divergence patterns (Jiang et al., 2007b; Kral and Eggermont, 2007; Ahissar et al., 2008). That is, dissimilar lower-level input representations can map to the same higher-level representations; and similar lower-level input representations can map to different higher-level representations.

\section{SPEECH PROCESSING PATHWAYS}

Reverse hierarchy theory has not, to our knowledge, previously been extended to an explicit theory of multisensory constraints on 
unisensory perceptual learning, but the evidence on the diversity and extent of cortical and subcortical multisensory connections (Foxe and Schroeder, 2005; Ghazanfar and Schroeder, 2006; Driver and Noesselt, 2008; Kayser et al., 2012) suggests that higher-level representations in one sensory-perceptual system can be used to gain access to lower-level representations in another sensoryperceptual system. Figure $\mathbf{6}$ is a schematic view of auditory and visual speech processing pathways. It suggests that at each level of stimulus processing - basic features (e.g., spectrotemporal auditory features and spatiotemporal visual features not specific to speech), phonetic features (linguistically relevant sub-phonemic integrated basic features), phonemes (syllables or word forms, i.e., linguistically relevant categories) - there is the possibility of multisensory integrative processes and also unisensory representations. Various experimental results have been interpreted as evidence that visual speech information can converge as early as primary auditory cortex (e.g., Sams et al., 1991; Calvert et al., 1997; Giard and Peronnet, 1999; Möttönen et al., 2002; Raij et al., 2010), and anatomical animal studies have provided evidence of multisensory connectivity as low as primary visual and auditory areas (Ghazanfar et al., 2008; Falchier et al., 2012). Such results have been interpreted as support for early and obligatory multisensory integration (Rosenblum, 2008). Other findings point to multisensory integration at higher cortical levels, such as superior temporal sulcus, suggesting that extensive unisensory integration has occurred prior to integrative activity (Miller and D'Esposito, 2005; Hasson et al., 2007; Bernstein et al., 2008a; Nath and Beauchamp, 2011).

Figure 6 shows a parallel structure for unisensory auditory and visual speech processing. The parallel unisensory hierarchy for visual speech receives diverse support in the literature. For example, dissimilarity measures of visual speech stimuli significantly account for consonant perceptual dissimilarity (Jiang et al., 2007a; Files and Bernstein, in preparation). That is, physical

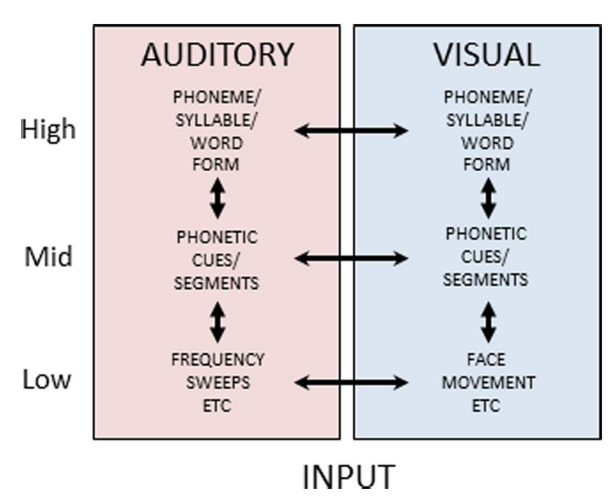

FIGURE 6 | Auditory and visual speech pathways. Figure 6 schematically depicts cortical processing pathways and their interactions for auditory and visual speech. Separate uni-sensory processing pathways are thought to exist for mapping from low-level stimulus features to high-level form-based representations of speech stimuli. During perceptual processing, information is thought to predominantly flow from low-to-high, however feedback pathways are available along both pathways. Additionally, at each level double arrowed lines between the pathways indicate the potential for multisensory integrative processing. optical measures can account for significant variance in visual perceptual identification and discrimination. Patterns of confusions for lipreading words are reliably accounted for by visual perception of spoken phonemes (Mattys et al., 2002). Visual perceptual confusions account for results on visual spoken word identifications better than auditory perceptual confusions (Auer, 2002). Visual speech mismatch negativity event-related potentials have been localized posterior to auditory temporal cortices (Ponton et al., 2009; Files and Bernstein, submitted), and visual speech processing has been localized with functional magnetic resonance imaging in posterior superior temporal cortex and adjacent middle temporal cortex, consistent with speech representation in the high-level vision pathway (Bernstein et al., 2011).

Thus, speech perception can be multisensory, visual-only, or auditory-only, and there is support for representations that correspond to these three possibilities. It also seems reasonable to conclude across the many results on speech perception involving auditory and visual stimuli that multisensory integration is available at every level of speech processing, consistent with a highly multisensory cerebral cortex (Ghazanfar and Schroeder, 2006). How could this diversity of integrative resources contribute to the discrepant results of Experiments 1 and 3 ?

\section{EXPLANATION FOR DIVERGENT MULTISENSORY TRAINING OUTCOMES}

In order to explain our divergent results, we need to focus on the level at which auditory perceptual learning took place. Our results point to phonetic features, which are linguistically relevant sub-phonemic representations that typically are said to map to phoneme categories (for discussion of features, Jakobson et al., 1961; Chomsky and Halle, 1968) but could also map directly to syllable, morpheme, or word-level categories (Grossberg et al., 1997; Vitevitch and Luce, 1999; Norris et al., 2000). The results point to auditory perceptual learning of phonetic features, because learning generalizes to forced-choice consonant identification in new words, and learning is differentially affected by the position of the consonant. If consonants were learned as unanalyzed units, we would not expect that their position in the word would be a significant effect in our results. The medial consonant affords the most phonetic feature information, which is obtained from the vowel transitions into and out of the consonant (Stevens, 1998), and therefore phonetic feature learning should result in more gains when feature information is richer. In addition, the largest amount of auditory learning was for the medial consonant position following AV training: Auditory perceptual learning was more sensitive to phonetic details in the auditory stimuli when the training was AV.

To be clear, phonetic features are integrated representations based on basic sound features. That phonetic features are complex combinations of information about the acoustic attributes of speech has been extensively researched (Stevens, 1998). For example, the place of articulation (e.g., involved in the distinction $/ \mathrm{b} /$ versus $/ \mathrm{d} /$ ) is instantiated in the acoustic signal partly by the center frequency and transitions of the speech formants (resonance of the vocal tract). The feature known as voicing (e.g., involved in the distinction /b/ versus /p/) is instantiated partly by 
the temporal offset difference between consonant initiation in the supralaryngeal vocal tract and the onset of glottal pulsing (Lisker et al., 1977). Relatively little research has been carried out on the neural bases of phonetic feature processing, with most speech perception research focused on levels either lower than or higher than phonetic features (Binder et al., 2000; Scott, 2005; Hickok and Poeppel, 2007; Liebenthal et al., 2010), however, Obleser and Eisner (2009) have identified a site of phonetic feature processing anterior to the primary auditory cortical areas in superior temporal gyrus. This gives support to the possibility of focused phonetic feature learning.

When speech is degraded or transformed, perceptual confusions among phonemes can be described in terms of loss of phonetic feature distinctions (Miller and Nicely, 1955; Wang and Bilger, 1973). The problem for auditory perceptual learning of vocoded speech is to remap available basic auditory features (such as frequency and temporal features) in the novel transformation to phonetic features that support the perception of syllables, morphemes, and/or words.

Figure 7 illustrates our proposed model for the outcomes of Experiments 1 and 3 within the context of multisensory and unisensory processing resources and the RHT of perceptual learning. In Figure 7, the blue and red circles represent visual and auditory phonetic speech features, respectively. For purposes here and in Figure 7, the category that phonetic features target is not important to define, because the results of the three experiments point to auditory perceptual learning at the phonetic feature level targeting phonemes, and as pointed out

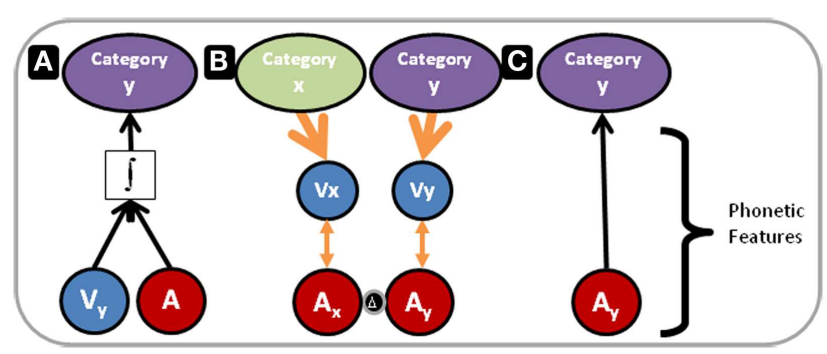

FIGURE 7 | Perceptual learning versus integration model. The blue and red circles in the lower part of Figure 7 represent visual and auditory phonetic speech features, respectively. These correspond to the mid level of processing in Figure 6. The categories at the top of the figure correspond to representations at the high-level of processing in Figure 6.

(A) Depicts processing under conditions in which acoustic phonetic features alone are not sufficient to specify the phoneme category. The integrated audiovisual phonetic features do provide adequate information. Perceptual processing flows bottom-up, and remapping along the auditory pathway has not occurred. In contrast, (B) Depicts a reverse flow of information. As in (A), Combined audiovisual information is sufficient to specify phoneme categories (not shown). However, here a reverse search is initiated. Higher-level visual speech categories, $x$ and $y$, feed back to visual phonetic features, $V x$ and $V y$, that use natural audiovisual correlations (orange double arrowed lines) to guide the search for relevant distinctions in acoustic-phonetic feature representations. The two red circles separated by a delta are labeled $A x$ and $A y$ because the acoustic phonetic features are now distinct. (C) Depicts auditory-only processing, following the perceptual learning depicted in (B). The acoustic phonetic features alone are now sufficient to specify the phoneme category. above features could target phonemes, syllables, morphemes, or words.

In Figure 7A, vocoding has removed or distorted the basic auditory information that is typically mapped to phonetic features of natural speech. The phonetic feature level is inadequate to specify the phoneme category (phoneme categories for purposes here). But the visual speech information provides the needed phonetic information (Summerfield, 1987), the information is integrated, and the perceptual task is carried out at an immediate high-level of perception, as predicted by RHT. However, with early integration the perceptual task can be accomplished without scrutiny of auditory lower-level representations, and if the visual stimulus is unavailable performance drops. This is our explanation for the finding in Experiment 3, in which performance following $\mathrm{AV}$ training was lower than following $\mathrm{AO}$ training.

Several factors in Experiment 3 could have reduced the likelihood that participants focused on the auditory information when the training was AV. RHT predicts that when semantic processing is required, low-level access is precluded (Ahissar et al., 2008; Nahum et al., 2008). In Experiment 3, participants were trained to criterion, and they were free to train on as many lists as possible during a training session. Trying to learn more than one list in a day could have directed attention to semantic relationships. Training to criterion on more than one list could have encouraged less attention to the auditory input, because it might have led participants to put a premium on the rate at which the paired associations were learned rather than on the accuracy of the AO tests. Also, given that perception of AV speech stimuli is frequently faster and more reliable (Sumby and Pollack, 1954; Van Wassenhove et al., 2005; Ross et al., 2007), we surmise that in Experiment 3 the perceived effort to learn the paired associations was lower under AV versus AO conditions. This perceived reduced effort might have also favored relying on high-level representations that were fed by AV integration. While it is true that semantic category training can result in retuning representations (Jiang et al., 2007b) and change in sensitivity to category boundaries (Goldstone, 1994), such training typically involves less diverse stimuli than the ones in the present study.

Figure 7B has two columns. Each has a downward arrow from a higher-level of visual speech category representation to a level that is correlated with auditory representations. Remapping from basic sound to phonetic features has taken place due to top-down guidance within the visual system. The red circles are labeled $A x$ and $A y$, because phonetic features are now distinct. We think that the auditory distinctions that were learned in our study must be readily available at the level of basic features (not indicated in Figure 7), because learning was relatively fast and low-level auditory retuning is likely not affected over such a brief period (Kral and Eggermont, 2007). Likewise, the rapid learning argues against learning based on new connections via dendritic growth and arborization.

We hypothesize that this remapping process makes use of natural correlations between auditory and visual speech stimuli, indicated in Figure 7B with the double pointed arrows. These natural AV correlations provides a link whereby visual information can help guide attention to the relevant distinctions in the 
auditory representations. Research on the predictability of acoustic signals from optical signals and vice versa has shown that there are high-levels of correlation between acoustic and optical speech signals (Yehia et al., 1998; Jiang et al., 2002; Jiang and Bernstein, 2011). Perceptual evidence shows that quantified correlation of the physical acoustic and optical speech signals can account for AV speech responses with matched and mismatched (McGurk type) stimuli (Jiang and Bernstein, 2011). Visual speech stimuli have been suggested to modify auditory speech processing through modulatory effects on neuronal excitability (Schroeder et al., 2008). Speech-in-noise experiments suggest that perceivers adjust their perception and neural networks change in relationship to the relative reliability of auditory or visual information (Ross et al., 2007; Nath and Beauchamp, 2011), or the temporal alignment of the stimuli (Miller and D'Esposito, 2005). We are suggesting that top-down processing from visual speech representations can guide access to distinctive auditory features that can be remapped to phonetic features for novel speech transformations. Top-down guidance via orthographic representations has been suggested as another basis for auditory perceptual learning of vocoded speech (Davis et al., 2005).These two types of top-down guidance might result in different learning. Specifically, the multisensory speech correlations might provide more fine-grained guidance for phonetic learning than orthography.

In Figure 7C, following the successful remapping, when $\mathrm{AO}$ stimuli are presented, the auditory mapping to the category is sufficient to carry out the task. Figure 7C corresponds to the result in Experiment 1 that AV PA training was more effective than AO training or merely re-testing in Experiment 2.

\section{SOME IMPLICATIONS FOR TRAINING}

Results reported here could be important clinically, for example, to crafting strategies for patients newly fitted with a cochlear implant (Zeng et al., 2004). The goal of such training is to assist the cochlear implant user in gaining access to the information in the degraded or impoverished signal delivered by the auditory prosthesis. Such patients can benefit from auditory training, but the benefits are typically not large (Fu et al., 2005; Stacey et al., 2010). A focus in training studies has been on which linguistic units such as phonological features, syllables, words, or sentences might best promote auditory perceptual learning (Fu et al., 2005; Stacey et al., 2010). However, the goals of training might be better served by focusing on the flow of information processing, specifically, the possibility that reverse hierarchy processing is needed to gain access to the available information (Kral and Eggermont, 2007; Auer and Bernstein, 2012). Focus is needed on the possibility that top-down guidance must be crafted that allows access to the level of representation where additional cues are available to be remapped. The current results support this view. But knowledge is also needed to predict when $\mathrm{AV}$ integration can impede auditory perceptual learning.

The results here are particularly relevant to training young cochlear implanted children who have not yet learned to read. In contrast to literate normal-hearing adults who can use orthographic representations or clear speech to guide perceptual learning (Davis et al., 2005; Hervais-Adelman et al., 2011), children's guides are often limited to multisensory information delivered via lipreading, visual signed language or fingerspelling, and/or vibrotactile speech displays (Bernstein et al., 1991; Auer et al., 1998).

A concerted effort was made in the twentieth century to design and test vibrotactile speech perception prostheses to supplement lipreading by deaf individuals including children. While the intent of the research was to learn how to convey speech through mechanical vibration signals, combined visual-vibrotactile training was shown to be associated with improved visual-only speech perception (Boothroyd and Hnath-Chisolm, 1988; Eberhardt et al., 1990; Bernstein et al., 1991; Kishon-Rabin et al., 1996). These improvements in lipreading sometimes exceeded the vibrotactile learning. This type of result suggests that when a novel speech signal is combined with a more familiar one, attention might be directed toward discerning additional information from the more familiar signal rather than the target novel signal. Indeed, in a companion study (in preparation) to this one on prelingually deaf adults who obtained cochlear implants as adults, we found that $\mathrm{AV}$ training resulted in faster PA learning but poorer auditory-only test scores, consistent with attention to and reliance on the more familiar visual stimuli. Indeed, there is evidence that visual perceptual abilities and multisensory integration are affected by cochlear implant usage in adults (Rouger et al., 2007). Understanding is needed for how to devise training that uses multisensory stimuli to guide unisensory perceptual learning, rather than only effecting immediate high-level perception with concomitant failure to achieve discernment of available low-level distinctions.

\section{SUMMARY AND CONCLUSION}

In summary, the results reported here do not fall under the rubrics of faster or more accurate $\mathrm{AV}$ versus $\mathrm{AO}$ speech perception, effects that have been well-documented (e.g., Sumby and Pollack, 1954; Bernstein et al., 2004; Van Wassenhove et al., 2005; Ross et al., 2007). They concern AV versus AO training effects on auditory-only perceptual learning. The information in a visual speech stimulus, presented in synchrony with a correlated but degraded auditory stimulus, can be effective in promoting auditory speech perceptual learning of the degraded stimuli. The visual information can promote more learning than the auditory stimuli alone, because of the correlations between auditory and visual features or cues, and because top-down visual processes can guide access to available but unused auditory cues. However, the multisensory speech stimuli typically are more informative and easier to perceive, and multisensory perception can rely on integrated representations, thereby possibly impeding unisensory perceptual learning. Research is needed on what perceptual learning procedures are required so that multisensory stimuli can be used reliably to enhance unisensory perceptual learning.

\section{ACKNOWLEDGMENTS}

We thank our test subjects for their participation and our technicians for supporting the data acquisition. Research supported by NIH/NIDCD DC008308. 


\section{REFERENCES}

Ahissar, M., and Hochstein, S. (1997). Task difficulty and the specificity of perceptual learning. Nature 387, 401-406.

Ahissar, M., Nahum, M., Nelken, I., and Hochstein, S. (2008). Reverse hierarchies and sensory learning. Philos. Trans. R. Soc. Lond. B Biol. Sci. 364, 285-299.

Auer, E. T. Jr. (2002). The influence of the lexicon on speech read word recognition: contrasting segmental and lexical distinctiveness. Psychon. Bull. Rev. 9, 341-347.

Auer, E. T. Jr., and Bernstein, L. E. (1997). Speechreading and the structure of the lexicon: computationally modeling the effects of reduced phonetic distinctiveness on lexical uniqueness. J. Acoust. Soc. Am. 102, 3704-3710.

Auer, E. T. Jr., and Bernstein, L. E. (2012). "Plasticity for multisensory speech communication: evidence from deafness and normal hearing," in The New Handbook of Multisensory Processing, ed. B. E. Stein (Cambridge, MA: MIT), 453-466.

Auer, E.T. Jr., Bernstein, L. E., and Coulter, D. C. (1998). Temporal and spatio-temporal vibrotactile displays for voice fundamental frequency: an initial evaluation of a new vibrotactile speech perception aid with normal-hearing and hearing-impaired individuals. J. Acoust. Soc. Am. 104, 2477-2489.

Beauchamp, M. S., Lee, K. E., Argall, B. D., and Martin, A. (2004). Integration of auditory and visual information about objects in superior temporal sulcus. Neuron 41, 809-823.

Bernstein, L. E. (2012). "Visual speech perception," in AudioVisual Speech Processing, eds E. Vatikiotis-Bateson, G. Bailly, and P. Perrier (Cambridge: Cambridge University), 21-39.

Bernstein, L. E., Auer, E. T. Jr., and Takayanagi, S. (2004). Auditory speech detection in noise enhanced by lipreading. Speech Commun. 44, 5-18.

Bernstein, L. E., Auer, E. T. Jr., Wagner, M., and Ponton, C. W. (2008a). Spatio-temporal dynamics of audiovisual speech processing. Neuroimage 39, 423-435.

Bernstein, L. E., Lu, Z. L., and Jiang, J. (2008b). Quantified acoustic-optical speech signal incongruity identifies cortical sites of audiovisual speech processing. Brain Res. 1242, 172-184.
Bernstein, L. E., Demorest, M. E., Coulter, D. C., and O'connell, M. P. (1991). Lipreading sentences with vibrotactile vocoders: performance of normal-hearing and hearingimpaired subjects. J. Acoust. Soc. Am. 90, 2971-2984.

Bernstein, L. E., Demorest, M. E., and Tucker, P. E. (2000). Speech perception without hearing. Percept. Psychophys. 62, 233-252.

Bernstein, L. E., Jiang, J., Pantazis, D., Lu, Z.-L., and Joshi, A. (2011). Visual phonetic processing localized using speech and nonspeech face gestures in video and pointlight displays. Hum. Brain Mapp. 32, 1660-1667.

Binder, J. R., Frost, J. A., Hammeke, T. A., Bellgowan, P. S., Springer, J. A., Kaufman, J. N., et al. (2000). Human temporal lobe activation by speech and nonspeech sounds. Cereb. Cortex 10, 512-528.

Boothroyd, A., and Hnath-Chisolm, T. (1988). Spatial, tactile presentation of voice fundamental frequency as a supplement to lipreading: results of extended training with a single subject. J. Rehabil. Res. Dev. 25, 51-56.

Calvert, G. A., Bullmore, E. T., Brammer, M. J., Campbell, R., Williams, S. C., McGuire, P. K., et al. (1997). Activation of auditory cortex during silent lipreading. Science 276, 593-596.

Calvert, G. A., Campbell, R., and Brammer, M. J. (2000). Evidence from functional magnetic resonance imaging of crossmodal binding in the human heteromodal cortex. Curr. Biol. 10, 649-657.

Chomsky, N., and Halle, M. (1968). The Sound Pattern of English. New York: Harper \& Row.

Davis, M. H., Johnsrude, I. S., HervaisAdelman, A., Taylor, K., and McGettigan, C. (2005). Lexical informatio drives perceptual learning of distorted speech: Evidence from the comprehension of noise-vocoded sentences. J. Exp. Psychol. Gen. 134, 222-241.

Driver, J., and Noesselt, T. (2008). Multisensory interplay reveals crossmodal influences on 'sensory-specific' brain regions, neural responses, and judgments. Neuron 57, 11-23.

Eberhardt, S. P., Bernstein, L. E., Demorest, M. E., and Goldstein, M. H. Jr. (1990). Speechreading sentences with single-channel vibrotactile presentation of voice fundamental frequency. J. Acoust. Soc. Am. 88, 1274-1285.
Falchier, A., Cappe, C., Barone, P., and Schroeder, C. E. (2012). "Sensory convergence in low-level cortices," in The New Handbook of Multisensory Processing, ed. B. E. Stein (Cambridge, MA: MIT), 67-79.

Falchier, A., Renaud, L., Barone, P., and Kennedy, H. (2001). Extensive projections from the primary auditory cortex and polysensory area STP to peripheral area V1 in the macaque. Abstr. Soc. Neurosci. 27.

Felleman, D. J., and Van Essen, D. C. (1991). Distributed hierarchical processing in the primate cerebral cortex. Cereb. Cortex 1, 1-47.

Foxe, J. J., and Schroeder, C. E. (2005). The case for feedforward multisensory convergence during early cortical processing. Neuroreport 16, 419-423.

Fu, Q.-J., Galvin, J., Wang, X., and Nogaki, G. (2005). Moderate auditory training can improve speech performance of adult cochlear implant patients. Acoust. Res. Lett. Online 6 , 106-111.

Ghazanfar, A. A., Chandrasekaran, C. and Logothetis, N. K. (2008). Interactions between the superior temporal sulcus and auditory cortex mediate dynamic face/voice integration in Rhesus monkeys. J. Neurosci. 28, 4457-4469.

Ghazanfar, A. A., Maier, J. X., Hoffman, K. L., and Logothetis, N. K. (2005). Multisensory integration of dynamic faces and voices in rhesus monkey auditory cortex. J. Neurosci. 25, 5004-5012.

Ghazanfar, A. A., and Schroeder, C. E. (2006). Is neocortex essentially multisensory? Trends Cogn. Sci. (Regul. Ed.) 10, 278-285.

Giard, M. H., and Peronnet, F. (1999). Auditory-visual integration during multimodal object recognition in humans: a behavioral and electrophysiological study. J. Cogn. Neurosci. 11, 473-490.

Goldstone, R. L. (1994). Influences of categorization on perceptual discrimination. J. Exp. Psychol. Hum. Percept. Perform. 123, 178-200.

Goldstone, R. L. (1998). Perceptual learning. Annu. Rev. Psychol. 49, 585-612.

Green, K. P., and Kuhl, P. K. (1989) The role of visual information in the processing of place and manner features in speech perception. Percept. Psychophys. 45, 34-42.
Grossberg, S., Boardman, I., and Cohen, M. (1997). Neural dynamics of variable-rate speech categorization. J. Exp. Psychol. Hum. Percept. Perform. 23, 483-503.

Hasson, U., Skipper, J. I., Nusbaum, H. C., and Small, S L. (2007). Abstract coding of audiovisual speech: beyond sensory representation. Neuron 56, 1116-1126.

Hazan, V., Sennema, A., Faulkner, A., and Ortega-Llebaria, M. (2006). The use of visual cues in the percpetion of non-native consonant contrasts. J. Acoust. Soc. Am. 119, 1740-1751.

Hervais-Adelman, A., Davis, M. H., Johnsrude, I. S., Taylor, K. J., and Carlyon, R. P. (2011). Generalization of perceptual learning of vocoded speech. J. Exp. Psychol. Hum. Percept. Perform. 37, 293-295.

Hickok, G., and Poeppel, D. (2007). The cortical organization of speech processing. Nat. Rev. Neurosci. 8, 393-402.

Hubel, D. H., and Wiesel, T. N. (1962). Receptive fields, binocular interaction and functional architecture in the cat's visual cortex. J. Physiol. 160, 106-154.

Iverson, P., Bernstein, L. E., and Auer, E. T. Jr. (1998). Modeling the interaction of phonemic intelligibility and lexical structure in audiovisual word recognition. Speech Commun. 26, 45-63.

Jakobson, R., Fant, C. G. M., and Halle, M. (1961). Preliminaries to Speech Analysis: The Distinctive Features and their Correlates. Cambridge, MA: MIT.

Jiang, J., Alwan, A., Keating, P., Auer, E. T. Jr., and Bernstein, L. E. (2002). On the relationship between face movements, tongue movements, and speech acoustics. EURASIP J. Appl. Signal Processing 2002, 1174-1188.

Jiang, J., Auer, E. T. Jr., Alwan, A., Keating, P. A., and Bernstein, L. E. (2007a). Similarity structure in visual speech perception and optical phonetics. Percept. Psychophys. 69, 1070-1083.

Jiang, X., Bradley, E. D., Rini, R. A., Zeffiro, T., Vanmeter, J., and Riesenhuber, M. (2007b). Categorization training results in shape- and category-selective human neural plasticity. Neuron 53 , 891-903.

Jiang, J., and Bernstein, L. E. (2011). Psychophysics of the McGurk and other audiovisual speech integration effects. J. Exp. Psychol. 
Hum. Percept. Perform. 37, Mattys, S. L., Bernstein, L. E., and Auer, 1193-1209.

Kaas, J. H., and Hackett, T. A. (2000). Subdivisions of auditory cortex and processing streams in primates. Proc. Natl. Acad. Sci. U.S.A. 97, 11793-11799.

Kayser, C., Petkov, C. I., and Logothetis, N. K. (2009). Multisensory interactions in primate auditory cortex: fMRI and electrophysiology. Hear. Res. 258, 80-88.

Kayser, C., Petkov, C. I., Remedios, R., and Logothetis, N. K. (2012). "Multisensory influences on auditory processing: perspectives from $\mathrm{AMRI}$ and electrophysiology," in The Neural Bases of Multisensory Processes, eds M. M. Murray and M. T. Wallace (Boca Raton, FL: CRC), 99-113.

Kishon-Rabin, L., Boothroyd, A., and Hanin, L. (1996). Speechreading enhancement: a comparison of spatial-tactile display of voice fundamental frequency (F0) with auditory F0. J. Acoust. Soc. Am. 100, 593-602.

Kral, A., and Eggermont, J. J. (2007). What's to lose and what's to learn: development under auditory deprivation, cochlear implants and limits of cortical plasticity. Brain Res. Rev. $56,259-269$.

Liberman, A. M., Cooper, F. S., Shankweiler, D. P., and StuddertKennedy, M. (1967). Perception of the speech code. Psychol. Rev. 74, 431-461.

Liebenthal, E., Desai, R., Ellingson, M. M., Ramachandran, B., Desai, A., and Binder, J. R. (2010). Specialization along the left superior temporal sulcus for auditory categorization. Cereb. Cortex 20, 2958-2970.

Lisker, L., Liberman, A. M., Erickson, D. M., Dechovitz, D., and Mandler, R. (1977). On pushing the voice onset-time (VOT) boundary about. Lang. Speech 20, 209-216.

Logothetis, N. K., and Sheinberg, D. L. (1996). Visual object recognition. Annu. Rev. Neurosci. 19 , 577-621.

Ma, W. J., Zhou, X., Ross, L. A., Foxe, J. J., and Parra, L. C. (2009). Lip-reading aids word recognition most in moderate noise: a Bayesian explanation using high-dimensional feature space. PLoS ONE 4:e4638. doi:10.1371/journal.pone.0004638.

MacLeod, A., and Summerfield, Q. (1987). Quantifying the contribution of vision to speech perception in noise. Br. J. Audiol. 21, 131-141. E. T. Jr. (2002). Stimulus-based lexical distinctiveness as a general word-recognition mechanism. Percept. Psychophys. 64, 667-679.

McGurk, H., and MacDonald, J. (1976). Hearing lips and seeing voices. Nature 264, 746-748.

Miller, G. A., and Nicely, P. E. (1955). An analysis of perceptual confusions among some English consonants. J. Acoust. Soc. Am. 27, 301-315.

Miller, L. M., and D'Esposito, M. (2005). Perceptual fusion and stimulus coincidence in the cross-modal integration of speech. J. Neurosci. 25, 5884-5893.

Möttönen, R., Krause, C. M., Tiippana, K., and Sams, M. (2002). Processing of changes in visual speech in the human auditory cortex. Cogn. Brain Res. 13, 417-425.

Nahum, M., Nelken, I., and Ahissar, M. (2008). Low-level information and high-level perception: the case of speech in noise. PLoS Biol. 6:e126. doi:10.1371/journal.pbio.0060126.

Nath, A. R., and Beauchamp, M. S. (2011). Dynamic changes in superior temporal sulcus connectivity during perception of noisy audiovisual speech. J. Neurosci. 31, 1704-1714.

Nath, A. R., and Beauchamp, M. S. (2012). A neural basis for interindividual differences in the McGurk effect, a multisensory speech illusion. Neuroimage 59, 781-787.

Norris, D., McQueen, J. M., and Cutler, A. (2000). Merging information in speech recognition: feedback is never necessary. Behav. Brain Sci. 23, 299-370.

Obleser, J., and Eisner, F. (2009). Pre-lexical abstraction of speech in the auditory cortex. Trends Cogn. Sci. (Regul. Ed.) 31, 14-19.

Ponton, C. W., Bernstein, L. E., and Auer, E. T. Jr. (2009). Mismatch negativity with visual-only and audiovisual speech. Brain Topogr. 21, 207-215.

Raij, T., Ahveninen, J., Lin, F. H., Witzel, T., Jaaskelainen, B. L., Israeli, E., et al. (2010). Onset timing of cross-sensory activations and multisensory interactions in auditory and visual sensory cortices. Eur. J. Neurosci. 31, 1772-1782.

Reisberg, D., McLean, J., and Goldfield, A. (1987). "Easy to hear but hard to understand: a lipreading advantage with intact auditory stimuli," in Hearing by Eye: The Psychology of Lip-reading, eds B. Dodd and R. Campbell (London: Lawrence Erlbaum), 97-113.

Rosenblum, L. D. (2008). Speech perception as a multimodal phenomenon. Curr. Dir. Psychol. Sci. 17, 405-409.

Ross, L. A., Saint-Amour, D., Leavitt, V. N., Javitt, D. C., and Foxe, J. J. (2007). Do you see what i am saying? Exploring visual enhancement of speech comprehension in noisy environments. Cereb. Cortex 17, 1147-1153.

Rouger, J., Lagleyre, S., Fraysse, B., Deneve, S., Deguine, O., and Barone, P. (2007). Evidence that cochlear-implanted deaf patients are better multisensory integrators. Proc. Natl. Acad. Sci. U.S.A. 104, 7295-7300.

Saint-Amour, D., De Sanctis, P., Molholm, S., Ritter, W., and Foxe, J. J. (2007). Seeing voices: High-density electrical mapping and sourceanalysis of the multisensory mismatch negativity evoked during the McGurk illusion. Neuropsychologia 45, 587-597.

Sams, M., Aulanko, R., Hamalainen, M., Hari, R., Lounasmaa, O. V., Lu, S. T., et al. (1991). Seeing speech: visual information from lip movements modifies activity in the human auditory cortex. Neurosci. Lett. 127, 141-145.

Schroeder, C. E., and Foxe, J. J. (2002). The timing and laminar profile of converging inputs to multisensory areas of the macaque neocortex. Cogn. Brain Res. 14, 187-198.

Schroeder, C. E., Lakatos, P., Kajikawa, Y., Partan, S., and Puce, A. (2008). Neuronal oscillations and visual amplification of speech. Trends Cogn. Sci. (Regul. Ed.) 12, 106-113.

Scott, S. K. (2005). Auditory processing - speech, space and auditory objects. Curr. Opin. Neurobiol. 15, 197-201.

Scott, S. K., Blank, C. C., Rosen, S., and Wise, R. J. (2000). Identification of a pathway for intelligible speech in the left temporal lobe. Brain 123(Pt 12), 2400-2406.

Scott, S. K., Rosen, S., Lang, H., and Wise, R. J. (2006). Neural correlates of intelligibility in speech investigated with noise vocoded speech a positron emission tomography study. J. Acoust. Soc. Am. 120, 1075-1083.
Seitz, P. F., Bernstein, L. E., Auer, E. T. Jr., and Maceachern, M. (1998). PhLex (Phonologically Transformable Lexicon): A 35,000-word Computer Readable Pronouncing American English Lexicon on Structural Principles, with Accompanying Phonological Transformations, and Word Frequencies. [Online]. Los Angeles: Copyright House Ear Institute. [Accessed].

Sekiyama, K., and Tohkura, Y. (1991). McGurk effect in non-english listeners: few visual effects for Japanese subjects hearing Japanese syllables of high auditory intelligibility. J. Acoust. Soc. Am. 90, 1797-1805.

Skipper, J. I., Van Wassenhove, V., Nusbaum, H. C., and Small, S. L. (2007). Hearing lips and seeing voices: how cortical areas supporting speech production mediate audiovisual speech perception. Cereb. Cortex 17, 2387-2399.

Stacey, P. C., Raine, C. H., O'donoghue, G. M., Tapper, L., and Twomey, T. (2010). Effectiveness of computerbased auditory training for adult users of cochlear implants. Int. $J$. Audiol. 49, 347-356.

Stevens, K. N. (1998). Acoustic Phonetics. Cambridge, MA: MIT Press.

Sumby, W. H., and Pollack, I. (1954). Visual contribution to speech intelligibility in noise. J. Acoust. Soc. Am. 26, 212-215.

Summerfield, A. Q. (1987). "Some preliminaries to a comprehensive account of audio-visual speech perception," in Hearing by Eye: The Psychology of Lip-Reading, eds B. Dodd and R. Campbell (London: Lawrence Erlbaum Associates, Inc.), 3-52.

Ungerleider, L. G., and Haxby, J. V. (1994). 'What' and 'where' in the human brain. Curr. Opin. Neurobiol. 4, 157-165.

Van Wassenhove, V., Grant, K. W., and Poeppel, D. (2005). Visual speech speeds up the neural processing of auditory speech. Proc. Natl. Acad. Sci. U.S.A. 102, 1181-1186.

Vitevitch, M. S., and Luce, P. A. (1999). Probabilistic phonotactics and neighborhood activation in spoken word recognition. J. Mem. Lang. 40, 374-408.

Wang, M. D., and Bilger, R. C. (1973). Consonant confusions in noise: a study of perceptual features. J. Acoust. Soc. Am. 54, 1248-1266. 
Williams, P., and Simons, D. (2000). Detecting changes in novel, complex threedimensional objects. Vis. cogn. 7, 297-322.

Yehia, H., Rubin, P., and VatikiotisBateson, E. (1998). Quantitative association of vocal-tract and facial behavior. Speech Commun. 26, 23-43.

Zeki, S. (2005). The Ferrier lecture 1995: behind the seen: the functional specialization of the brain in space and time. Philos. Trans. R. Soc. Lond. B Biol. Sci. 360, 1145-1183.

Zeng, F.-G., Popper, A. N., and Fay, R. R. (2004). Cochlear Implants: Auditory Prostheses and Electrical Hearing. New York: Springer.

Conflict of Interest Statement: The authors declare that the research was conducted in the absence of any commercial or financial relationships that could be construed as a potential conflict of interest.

Received: 04 December 2012; accepted: 28 February 2013; published online: 18 March 2013.

Citation: Bernstein LE, Auer ET, Eberhardt SP and Jiang J (2013) Auditory perceptual learning for speech perception can be enhanced by audiovisual training. Front. Neurosci. 7:34. doi: 10.3389/fnins.2013.00034
This article was submitted to Frontiers in Auditory Cognitive Neuroscience, a specialty of Frontiers in Neuroscience. Copyright (c) 2013 Bernstein, Auer, Eberhardt and Jiang. This is an openaccess article distributed under the terms of the Creative Commons Attribution License, which permits use, distribution and reproduction in other forums, provided the original authors and source are credited and subject to any copyright notices concerning any third-party graphics etc. 